\title{
Review of the ant genus Rogeria (Hymenoptera: Formicidae) in Guyana
}

\author{
JOHN S. LAPOLLA ${ }^{1} \&$ JEFFREY SOSA-CALVO ${ }^{1,2}$ \\ ${ }^{1}$ Department of Entomology, National Museum of Natural History, Smithsonian Institution, POB 37012, NHB, \\ CE518, MRC 188, Washington, D.C. 20013-7012. Present address: Department of Biological Sciences, \\ Towson University, 8000 York Road, Towson, Maryland 21252. E-mail: jlapolla@towson.edu \\ ${ }^{2}$ Maryland Center for Systematic Entomology, Department of Entomology, University of Maryland, 4112 Plant \\ Sciences Building, College Park, Maryland 20742. E-mail: sossajef@si.edu
}

\begin{abstract}
The myrmicine ant genus Rogeria in Guyana is reviewed. Using previously published records, older collections, and collections from recent leaf litter ant survey work, nine Rogeria species are now known from Guyana, with another two species that probably also occur in Guyana. Among these eleven species, a new species, $R$. tsumani, is here described, and four of them, including the new species, are new records for Guyana. A key to the species found in Guyana is provided, along with illustrations of all 11 species.
\end{abstract}

Keywords: biotic surveys, leaf litter, Myrmicinae, new species

\section{Introduction}

The rarely collected myrmicine ant genus Rogeria has a disjunct distribution, with 36 species previously known to occur in the New World and 3 species that are known from the Australasian region (Kugler 1994). Rogeria ants are cryptic in habit and specimens are primarily collected from leaf litter and rotten wood, usually through Berlese or Winkler sampling. Not much is known about their biology, except that nests have been taken in rotting logs, under rocks, and from the trunks of cacao trees (Kugler 1994).

Despite a recent revision of the genus by Kugler (1994), new species are still being discovered. Recent survey work in Guyana revealed a number of new records of Rogeria species for the country, along with the discovery of a new species collected by Neal Weber in 1936. Here we describe this new species and provide a review of the genus in Guyana. 
Specimens examined for this study are deposited in the following institutions:

CUIC Department of Entomology, Cornell University, Ithaca, New York, USA

LACM Los Angeles County Museum of Natural History, Los Angeles, California, USA

MCZC Museum of Comparative Zoology, Cambridge, Massachusetts, USA

UGBC Centre for the Study of Biological Diversity, University of Guyana, Georgetown, Guyana

USNM National Museum of Natural History, Washington, D.C., USA

Examination and measurement of specimens were completed at various magnifications using a light stereomicroscope (Leica Wild M10) and were recorded to the nearest $0.001 \mathrm{~mm}$. All measurements are given in millimeters, unless noted otherwise. Specimens were photographed using a JVC KY-F70B video camera mounted on a Leica M420 microscope and attached to an IBM Intellistation M Pro computer, on which composite images were assembled using Auto-Montage Version 3.04 software (Synoptics Ltd.). Morphological terminology employed throughout follows Bolton (1994) and Kugler (1994), with modifications where noted. Anatomical abbreviations are as follows:

CI Cephalic Index: (HW/HL) 100.

EL Eye Length: maximum diameter of compound eye in lateral view.

FLW Frontal Lobe Width: in full-face view, the maximum distance across frontal lobes.

GL Gaster Length: the length of the gaster in lateral view from the anteriormost point of first gastral segment (third abdominal segment) to the posteriormost point.

HL Head Length: the length of the head proper, excluding the mandibles; measured in full-face view from the midpoint of the anterior clypeal margin to a line drawn across the posterior margin from its highest points (to accommodate species where the posterior margin is concave).

HW Head Width: the maximum width of the head in full-face view (excluding the portion of the eyes that extends past the lateral margins of the head).

ML Mandible Length: in full face view, the distance from the anteriormost portion of head to apex of closed mandibles.

OI Ocular Index: (EL/HW) 100.

PL Petiole Length: in lateral view, the distance from the dorsal corner of the posterior peduncle to the nearest edge of the metapleural lobe (sensu Kugler 1994).

PPL Postpetiole Length: the maximum length of the postpetiole in lateral view.

PSI Propodeal Spine Index: (SpL/WL) 100.

PW Pronotal Width: the maximum width of the pronotum in dorsal view.

SI Scape Index: $(\mathrm{SL} / \mathrm{HW}) \bullet 100$.

SL Scape Length: the maximum length of the antennal scape excluding the condylar 
bulb.

SpL Propodeal Spine Length: the distance from the tip of the propodeal spine to the nearest border of the propodeal spiracle.

TL Total Length: HL+WL+PL+PPL+GL.

WL Weber's Length: in lateral view, the distance from the posteriormost border of the metapleural lobe to the anteriormost border of the pronotum, excluding the neck.

\section{Systematic treatment}

\section{Rogeria tsumani, NEW SPECIES}

Figs. 11 and 22

Holotype worker, GUYANA: Oronoque River just above juncture with New River, 29 July 1936 [coordinates determined by JSL: $2^{\circ} 45^{\prime} 00^{\prime \prime} \mathrm{N} 57^{\circ} 26^{\prime} 00^{\prime \prime} \mathrm{W}$ ], coll. N.A. Weber. Paratype worker same locality as holotype. Holotype in USNM, paratype in MCZC.

Diagnosis (worker): mesosoma smooth and shiny, with scattered short appressed hairs; propodeal spines long, slightly inclined posterad; petiole pedunculate and with a distinct node, convex in lateral view.

Description (worker). Head: subquadrate in dorsal view; dorsolateral surface of head with widely spaced punctures, smooth and shiny in between; dorsomedian surface of head weakly longitudinally carinulate; nuchal grooves very shallow, not visible in lateral view; mandibles triangular; inner margin with 5 teeth, apical tooth largest; clypeal apron with shallow median depression. Mesosoma: promesonotum faintly longitudinally carinulate; promesonotal suture weakly marked; mesopleural suture incomplete; anepisternum and metapleuron weakly rugose; katepisternum smooth and shiny; dorsum of propodeum transversely rugose; propodeal spines long $(\mathrm{SpL}=0.20-0.22)$ and slightly inclined posterad. Metasoma: petiole pedunculate, weakly rugulose, becoming smooth ventrally; petiolar ventral process smooth and shiny with a short anterior keel; postpetiole node tall, smooth and shiny; postpetiolar ventral margin rugose, with a short anterior keel. Body with scattered short, appressed hairs. Overall body color reddish-dark brown, with propodeum darker brown, and light brown legs. Queen and male unknown. Measurement: holotype and paratype $(\mathrm{N}=2): \mathrm{TL}=3.75-3.88, \mathrm{HL}=0.86-0.88, \mathrm{HW}=0.69-0.72, \mathrm{ML}=$ $0.39-0.41, \mathrm{SL}=0.52-0.55, \mathrm{EL}=0.12-0.15, \mathrm{PW}=0.55-0.55, \mathrm{WL}=0.95-0.97, \mathrm{SpL}=$ $0.20-0.22, \mathrm{PL}=0.44-0.48, \mathrm{FLW}=0.23-0.25, \mathrm{PPL}=0.22-0.23, \mathrm{GL}=0.89-0.89, \mathrm{CI}=$ $80-82, \mathrm{OI}=17-22, \mathrm{SI}=60-76, \mathrm{PSI}=21-23$.

Etymology: Named in honor of Dr. Ted Suman, Research Associate at the National Museum of Natural History. Without Ted's hard work and dedication to the Guyana Ant Project much of the research being conducted on the Guyana ant fauna would not be possible.

Natural history: The two known specimens of $R$. tsumani were collected from a rotten branch that fell off a tree from about 30 meters height as Weber was walking 

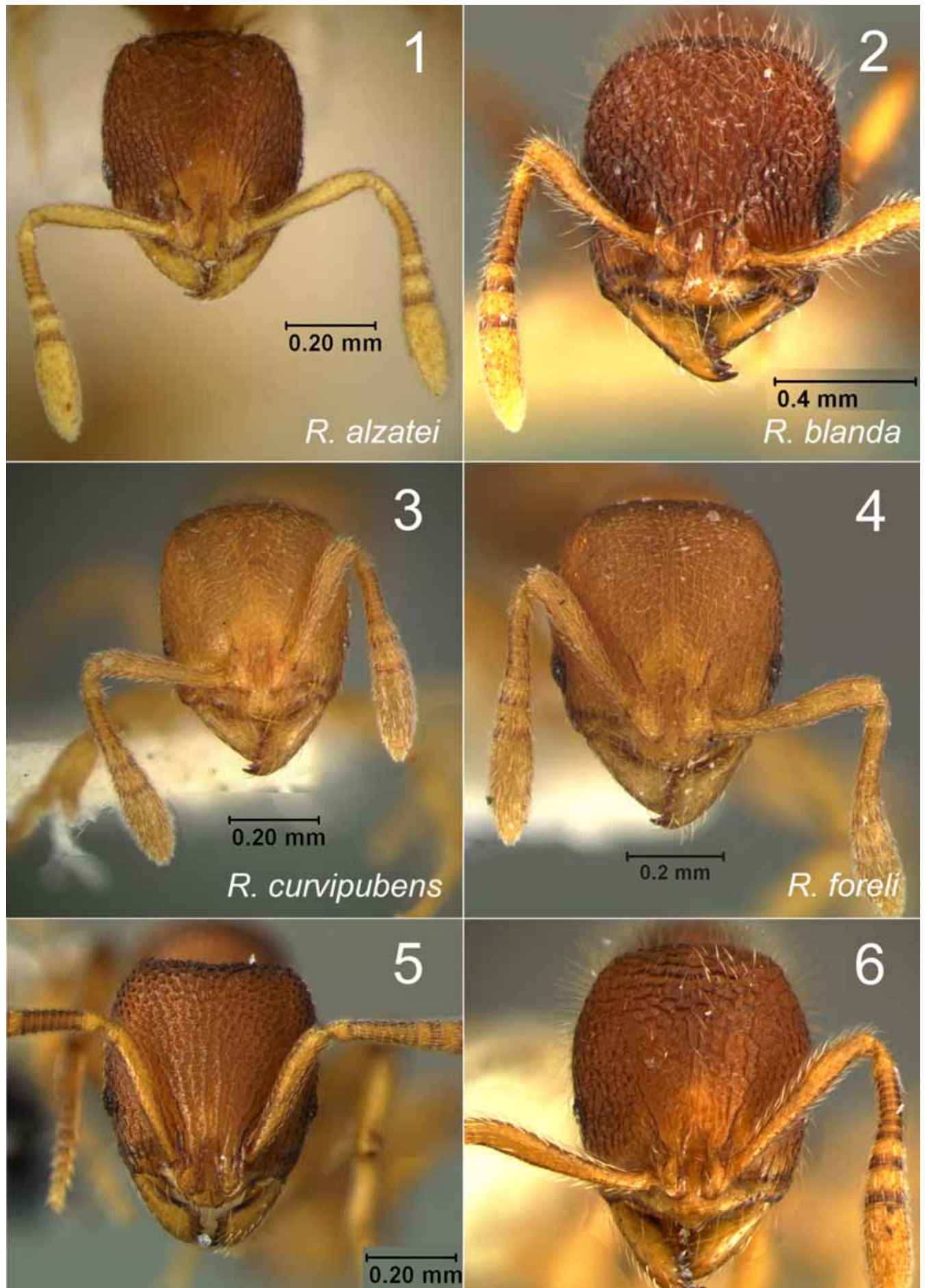

R. innotabilis

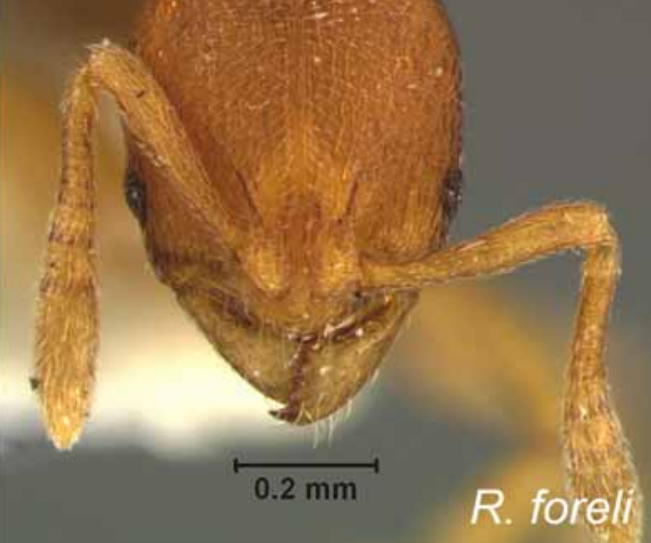

6

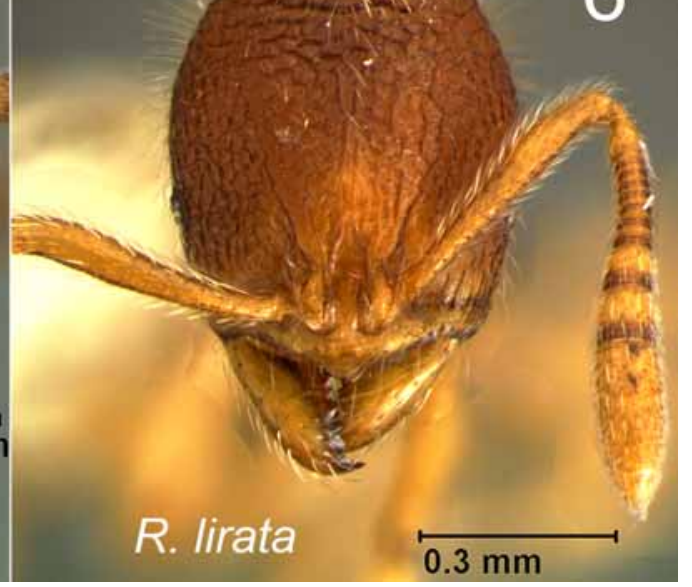

FIGURES 1-6. Rogeria species in full-face view. 


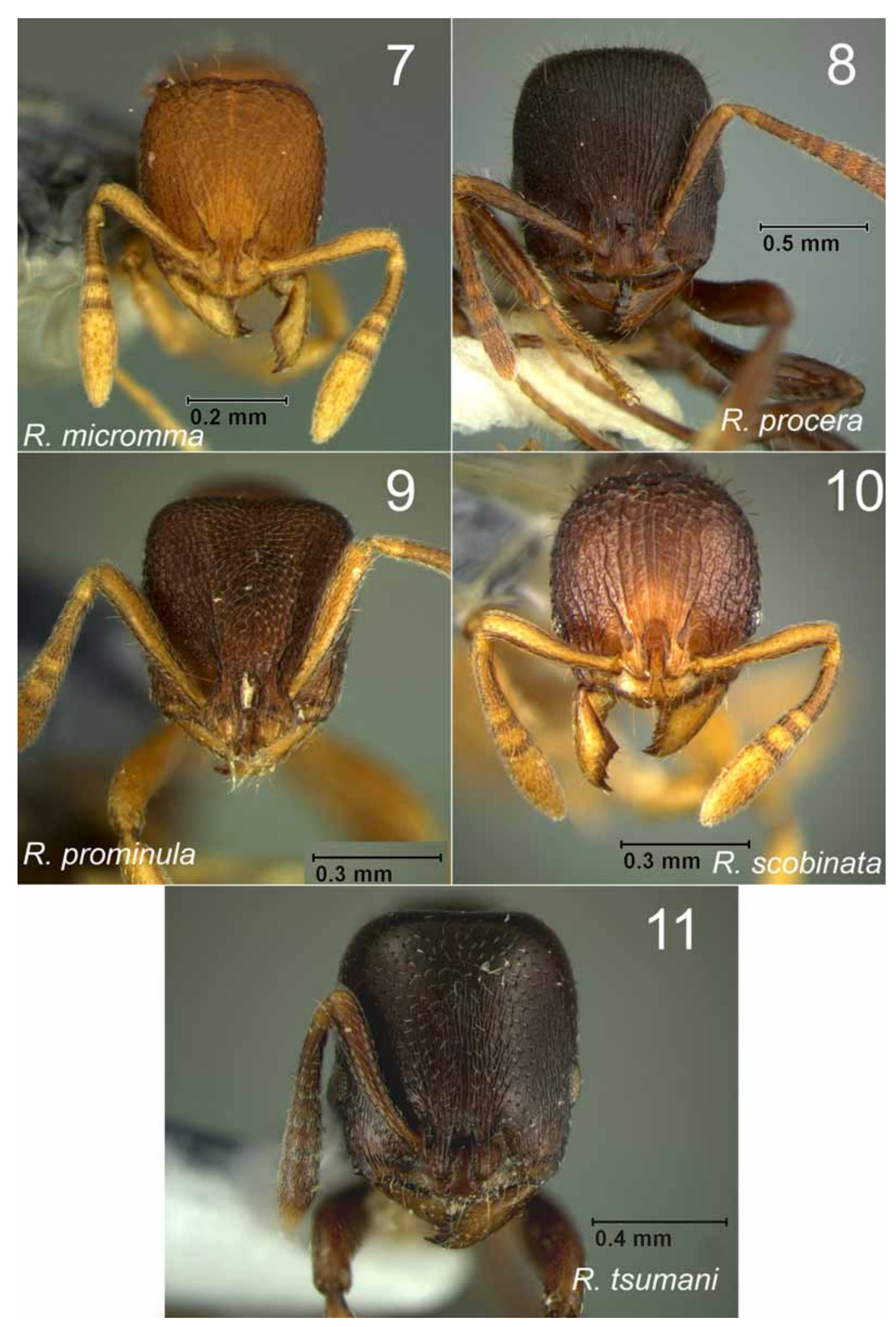

ZOOTAXA

1330

FIGURES 7-11. Rogeria species in full-face view. 


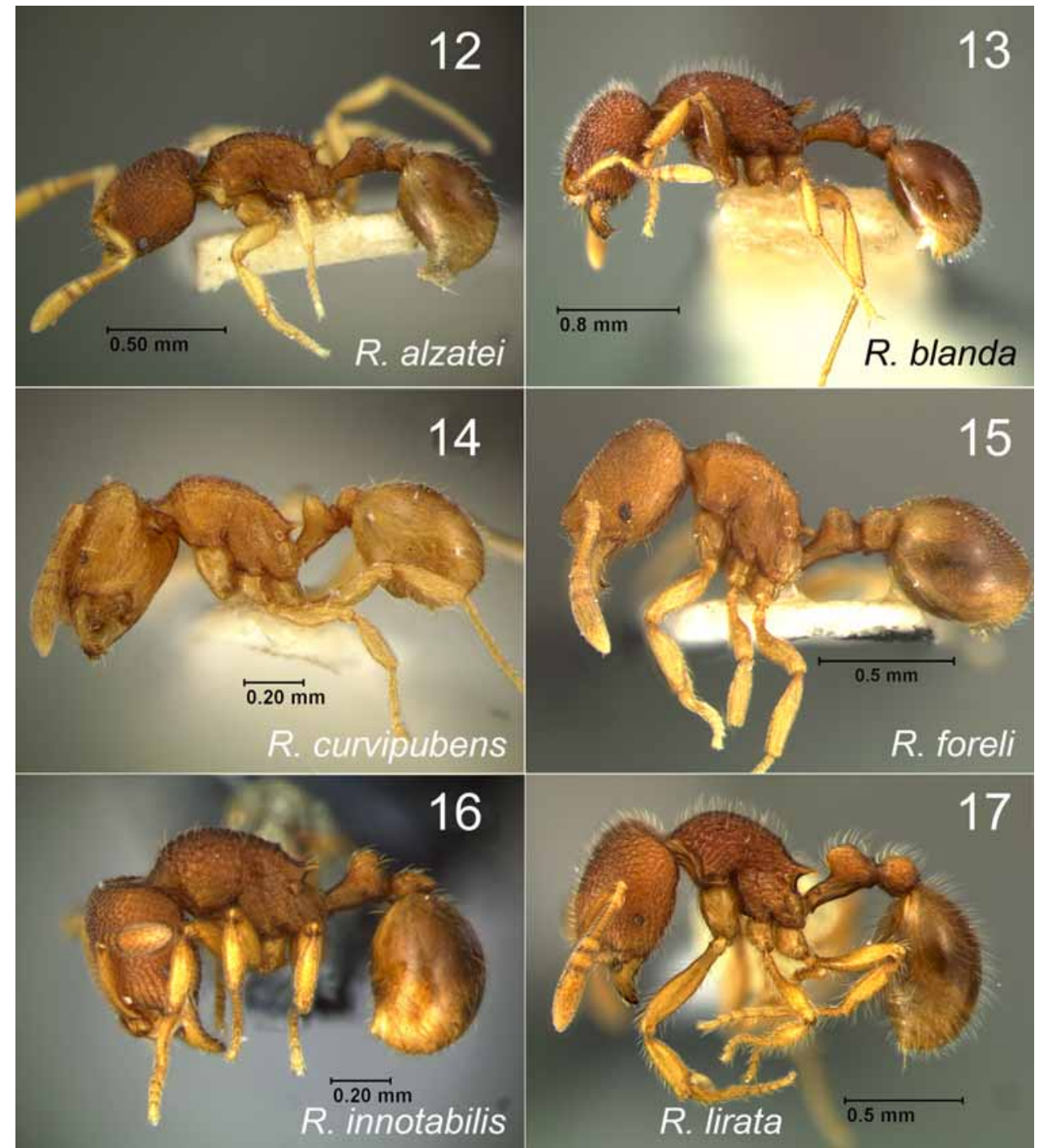

FIGURES 12-17. Rogeria species in lateral view.

through the forest (N.A. Weber, field notes, in MCZC archives). Weber reported looking through the rotting branch for the Rogeria nest, but he could not locate it. Rogeria tsumani is probably a canopy-dwelling species. While many Rogeria species are thought to nest in leaf litter and rotting wood on the ground, there is evidence that several species are arboreal or at least partly arboreal. Two species, $R$. blanda and $R$. tonduzi, have been taken from the trunks of cacao trees (Kugler 1994), and Longino reported $R$. blanda nesting in a standing dead tree trunk from La Selva, Costa Rica (Longino, 19 August 2005, http:// www.evergreen.edu/ants/genera/Rogeria/SPECIES/blanda/blanda.html). The trunk was small and broken off at the top, with very soft rotten wood. In addition, $R$. belti, R. foreli, $R$. scandens, and $R$. terescandens may possess arboreal habits. 

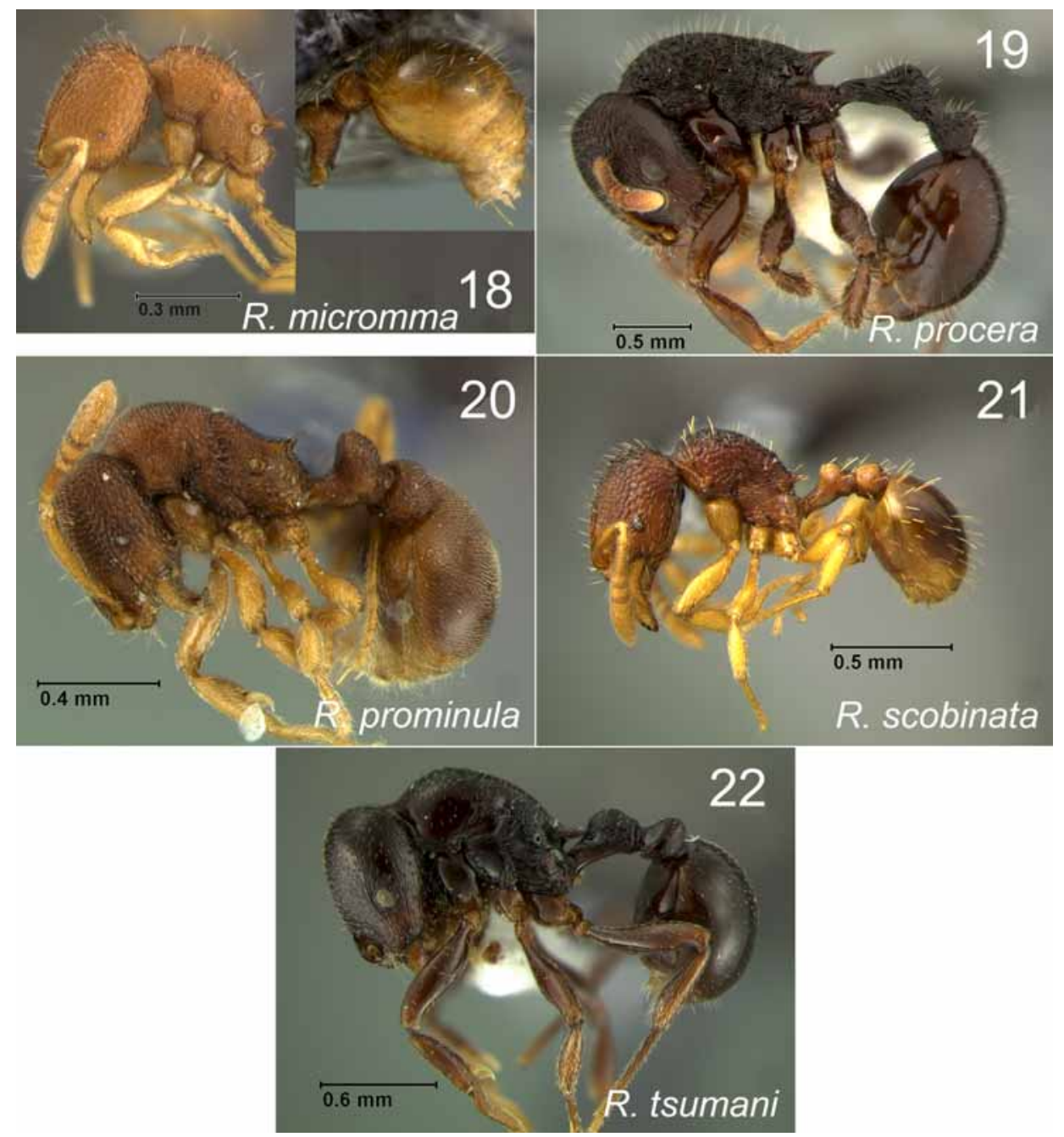

FIGURES 18-22. Rogeria species in lateral view.

Comments: Only four species of Rogeria are known in which the mesosomal dorsum bears only appressed hairs: $R$. bruchi, $R$. foreli, $R$. prominula, and $R$. tsumani. Both $R$. bruchi and $R$. foreli possess a much more erect and distinct petiolar node compared to $R$. tsumani, and the propodeal spines of $R$. bruchi and $R$. foreli are at roughly right angles to the longitudinal axis of the mesosoma. In $R$. tsumani, in contrast, the propodeal spines are roughly parallel to the longitudinal axis of the mesosoma. Rogeria prominula has a unique propodeal shape (fig. 20) and a rugoreticulate head and mesosoma. In Kugler's (1994) key, $R$. tsumani would key to R. bruchi. Rogeria tsumani is a larger species (TL 3.7-3.9 in $R$. tsumani vs. 2.2-2.6 in $R$. bruchi) with smaller eyes and darker brown coloration. Kugler (1994) constructed six informal species-groups. While he placed the majority of species into these species-groups, there was still a substantial number that he could not reliably place. Rogeria tsumani similarly fails to fall neatly into a species group. 
Rogeria alzatei (figs. 1 \& 12): known from Panama (type locality, Barro Colorado Island) to Paraguay, from Western Andes in Colombia to Eastern coast of Brazil. Also has been collected in Dominican Republic. In Guyana known from Oronoque River (Kugler 1994). Specimens have been collected outside of Guyana from Berlese and Winkler samples in moist forests to $1000 \mathrm{~m}$.

R. blanda (figs. 2 \& 13): known from Costa Rica to Southern Brazil. This species ranges from $50 \mathrm{~m}$ to $1000 \mathrm{~m}$ elevation. In Guyana known from Oko River (from Kugler 1994). In Costa Rica nests were found in the trunks of cacao tree, while in Peru a nest was found in a small rotten log that was suspended about $50 \mathrm{~cm}$ above the ground.

R. curvipubens (figs. 3 \& 14): known from some caribbean islands and inland from Mexico to Suriname. No known localities in Guyana, but it is known from Suriname (Tambahredjo; Sidoredjo; Dirkshoop; and La Poulle from Kugler 1994). This species has been collected from Berlese samples in tropical rain forest, successional forest, and dry tropical forest, below 500 m elevation.

$\boldsymbol{R}$. foreli (figs. $4 \&$ 15): this species has a disjunct distribution: the southwestern United States, Costa Rica, Panama, northern South America, and the Caribbean. There are no known localities in Guyana, but it is widespread in the northern Neotropics. Specimens have been collected from Berlese samples of leaf litter and rotten wood. In southeastern Arizona, specimens have been found under stones in oak-juniper woodland.

R. innotabilis" (figs. 5 \& 16): known from Mexico to northern Colombia. In Guyana known from Iwokrama Forest Reserve, Whitewater Camp, 4 43.890'N, 58 $50.992^{\prime} \mathrm{W}$,

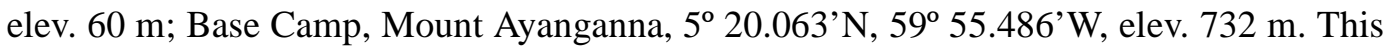
species has been collected in leaf litter.

$\boldsymbol{R}$. lirata (figs. 6 \& 17): this species is widespread throughout tropical South America. In Guyana this species was collected in winkler samples of leaf litter in Iwokrama Forest Reserve, Whitewater Camp, 4 43.890'N, 58 50.992'W, elev. $60 \mathrm{~m}$; Mabura Hill, $5^{\circ}$ 09.313'N, 58 41.982'W, elev. 64 m; Mazaruni River Forest Settlement (from Kugler 1994).

R. micromma $^{\#}$ (figs. $7 \&$ 18): this species was previously known only from several localities in Suriname. In Guyana it was collected in a lowland rainforest dominated by Chlorocardium (Lauraceae) trees (Mabura Hill, 5 09.313'N, 58 41.982'W, elev. 64 m.). 
The natural history of this species remains still unknown.

R. procera (figs. 8 \& 19): known from Guyana and Brazil. In Guyana this species has been collected in Oronoque River (from Kugler 1994). Nothing is known of the natural history of this species.

R. prominula (figs. 9 \& 20): known from its type locality: Manaus, Brazil. In Guyana this species is now known from Oronoque River (from N.A. Weber collection made in 1936). The natural history of this species remains unknown.

R. scobinata\# (figs. $10 \& 21$ ): this species ranges throughout much of tropical South America. In Guyana it was collected in Mabura Hill, 5 09.313'N, 58 ${ }^{\circ}$ 41.982'W, elev. 64 $\mathrm{m}$. Specimens have been collected from Berlese and Winkler samples of leaf litter and rotten wood in tropical forest below 1000 m elevation.

R. tsumani (figs. 11 \& 22): known only from the type locality in Guyana. See text for details of natural history.

\section{Key to Rogeria known from Guyana}

1 Hairs on mesosomal dorsum entirely appressed, or consisting of a mixture of mostly appressed and a few decumbent hairs

- Mesosomal dorsum bearing some erect hairs; appressed to suberect hairs may also be present

2 Body of clypeus not greatly projecting over clypeal apron (figs. 4 \& 11); color brown to shiny dark brown

- In lateral view, body of clypeus greatly projecting over clypeal apron (fig. 20); color dull brown prominula (figs. $9 \& 20$ )

3 Smaller species (TL: $1.9 \mathrm{~mm}-2.9 \mathrm{~mm}$ ); light brown to brown and dull. oreli (figs. 4 \& 15)

- $\quad$ Larger species (TL: $3.75 \mathrm{~mm}-3.88 \mathrm{~mm}$ ); dark brown and shiny..... tsumani (figs. 11 \& 22)

4 In lateral view, petiolar node low and curving gradually into anterior peduncle (figs. $13 \& 19)$

- In lateral view, petiolar node tall and more sharply differentiated from anterior peduncle, node rounded, subquadrate, or squamiform (figs. 12, 14, 16, 17, 18 \& 21)....

5 Dorsum of head rugoreticulate; area behind eyes rugoreticulate; reddish-brown 
- Dorsum of head with distinct longitudinal carinulae; area behind eyes smooth and shiny; dark brown. procera (figs. 8 \& 19)

6 Head surface uniformly rugoreticulate and/or areolate, no intervening shiny areas; hairs appressed to erect on head

- Head surface relatively smooth, only vaguely rugoreticulate or areolate, with intervening shiny areas; hairs appressed on head. curvipubens (figs. 3 \& 14)

7 Posterior portion of head rugoreticulate

- Posterior portion of head distinctly areolate innotabilis (figs. 5 \& 16)

8 Dorsal surfaces of head, mesosoma, and metasoma with sparse appressed and/or erect hairs

- Dorsal surfaces of head, mesosoma, and metasoma with a dense covering of erect hairs lirata (figs. 6 \& 17)

9 Lateral surface of mesosoma with distinct rugae; reddish-yellow

- Lateral surface of mesosoma without rugae, surface areolate; dull yellow micromma (figs. $7 \& 18$ )

10 Head largely with decumbent to appressed hairs; propodeal spines at roughly right angles relative to longitudinal axis of the mesosoma. alzatei (fig. 1 \& 12)

- Head largely with short erect hairs; propodeal spines nearly parallel to longitudinal axis of the mesosoma scobinata (figs. $10 \& 21$ )

\section{Acknowledgments}

We would like to thank Ted Schultz (National Museum of Natural History) for reviewing the manuscript and his continuing support of our research efforts. Gary Alpert (Harvard University) kindly provided us with the initial photographs of Rogeria procera. Roy Snelling (LACM) and James Liebherr (CUIC) kindly loaned us specimens. JSC was supported by a grant to T.R. Schultz (NSF IRCEB-011007). This project was funded by the Smithsonian Institution's Biological Diversity of the Guiana Shield Program and is number 107 in the BDG Program's publication series.

\section{References}

Bolton, B. (1994) Identification guide to the ant genera of the world. Harvard University Press, Cambridge, Mass., 222 pp.

Kugler, C. (1994) Revision of the ant genus Rogeria (Hymenoptera: Formicidae) with descriptions of the sting apparatus. Journal of Hymenoptera Research, 3, 17-89. 